\title{
FORMEN DES MODERNEN ANTISEMITISMUS UND PROBLEME SEINER ABWEHR*
}

Der moderne Antisemitismus beginnt nach einer Konvention der Historiker im Deutschen Kaiserreich um die Mitte der siebziger Jahre des neunzehnten Jahrhunderts. Als Anhaltspunkt für diese Datierung dient u.a. eine Serie von Artikeln in der deutschen Familienzeitschrift Die Gartenlaube: ihr Autor, Otto Glagau, beschuldigte die Juden, durch unehrliche Spekulationen den Sturz der Aktienpreise von 1873, der für ihn ein Börsenschwindel war, herbeigeführt zu haben. ${ }^{1}$

Meine Überlegungen werden sich - obwohl es Judenhaß und demgemäß Probleme seiner Abwehr bereits vorher gab - dieser Konvention anschlieBen und sich im wesentlichen auf Deutschland bzw. den deutschen Sprachbereich beschränken. Der Begriff Antisemitismus wurde hier 1879 von Wilhelm Marr zwar nicht erfunden, aber in die allgemeine Diskussion eingeführt: Marrs Buch, das noch im gleichen Jahr elf Auflagen erlebte, trug den bezeichnenden Titel Der Sieg des Judenthums über das Germanenthum. Vom nicht-confessionellen Standpunkt aus betrachtet. ${ }^{2} 1880$ gründete der protestantische preußische Hofprediger Stoecker seine Christlichsoziale Arbeiterpartei. Er benutzte Angriffe auf das Judentum und die Juden in seinen Berliner Versammlungsreden: er war ein eindrucksvoller Demagoge. Bis zum Ersten Weltkrieg entstanden danach eine Reihe weiterer Gruppen und Vereine, die antisemitische Ideen propagierten. Manche davon hatten nur ein einziges Ziel - den Antisemitismus. Andere nahmen antisemitische Forderungen in ihr Programm auf, ohne da $\beta$ der Judenhaß darin zentral stand. Dazu gehörte z.B. die Deutschkonservative Partei, die 1892 in ihrem sog. Tivoli-Programm nach einer Eindämmung des jüdischen

* Geringfügig überarbeiteter Text eines Vortrags, den ich am 20. Januar 1984 an der Gesamthochschule Bochum gehalten habe.

$1 \mathrm{Vgl}$. O. Glagau, Der Börsen- und Gründungs-Schwindel in Berlin. Gesammelte und stark vermehrte Artikel der ,,Gartenlaube”, Leipzig 1876.

2 W. Marr, Der Sieg des Judenthums über das Germanenthum. Vom nicht-confessionellen Standpunkt aus betrachtet, Bern 1879. 
Einflusses rief, aber auch der Bund der Landwirte, der Deutschnationale Handlungsgehilfenverband und der Alldeutsche Verband.

Ausgehend von diesen Vorgängen hat man versucht, den modernen Antisemitismus zu definieren. Charakteristisch sei, so wird erklärt, daß der Antisemitismus zur organisierten sozialen Bewegung geworden sei. Es wird ferner darauf hingewiesen, daß der Judenhaß nun auf den Widerruf der Emanzipation der Juden, d.h. ihrer rechtlichen Gleichstellung, gerichtet gewesen sei. Weiterhin wird auf den entschieden säkularen Charakter des Rassismus und der ihn stützenden Rassentheorie verwiesen.

Aber wie bei allen Definitionen komplexer sozialer Phänomene gibt es auch hier Bedenken. Die Beschränkung der Gleichheit der Juden, vor allem hinsichtlich von öffentlichen Ämtern und Funktionen, war seit dem Beginn der staatlichen Überlegungen zur Emanzipation - also in Preußen seit 1786 - ein Hauptgegenstand zahlloser Memoranden, Voten, Kommissionsbeschlüsse etc. Die preußischen Akten zur Judenpolitik von 1815 bis 1847, die z.Z. vom Leo Baeck Institute, New York, und der Historischen Kommission, Berlin, zur Veröffentlichung vorbereitet werden, enthalten mannigfaltiges Material hierzu. Ferner: es gab vor 1848 zwar noch keine politischen Parteien außerhalb der kirchlichen Gruppierungen, aber es gab soziale Bewegungen, auch antisemitische soziale Bewegungen. Man denke an 1819. Auch der Rassismus ist schon vor der Rassentheorie vorhanden. Eine seiner Komponenten - das negativ verzerrte Erscheinungsbild des Juden als Orientale, Semit etc. - existierte in der europäischen Tradition schon seit dem Mittelalter, in populären Bilderbogen und Flugblättern seit der Reformation.

Vollends sprengt der ideengeschichtliche Befund die Periodisierung. Man hat zu Recht darauf hingewiesen, daß die gehässige Denunzierung der jüdischen Religion als Nationalkult, die Kritik des Talmud, wie sie die gesamte antisemitische Literatur seit 1875 durchzieht, schon vorher den Judenhaß ideologisch motiviert hat. Auch die Aufklärung enthielt einen starken antijüdischen Strang. Der amerikanische Historiker Arthur Hertzberg hat dies u.a. für Voltaire gezeigt. ${ }^{3}$ Die französischen Frühsozialisten Fourier, Proudhon u.a. - identifizierten Judentum mit Kapitalismus, ebenso Karl Marx in seinem Aufsatz über Bruno Bauers Arbeiten zur Judenfrage. ${ }^{4}$ Und gerade der absolutistische Liberale Christian Wilhelm Dohm führte 1782 in die Emanzipationsdiskussion ein Motiv ein, in dem die Ablehnung von Judentum und Juden unverhüllt hervortrat: die Juden

\footnotetext{
3 A. Hertzberg, The French Enlightenment and the Jews, New York, London 1968.

${ }^{4}$ K. Marx, ,Zur Judenfrage", in: Deutsch-französische Jahrbücher, hrsg. von A. Ruge und K. Marx, Nr 1-2 (1844), S. 182-214; K. Marx, F. Engels. Gesamtausgabe, Erste Abteilung, Bd 2, Berlin 1982, S. 141-69.
} 
waren zu erziehen, sollten sich verbesserungsfähig zeigen. Soweit sich dies auf die auch von der jüdischen Geschichtsschreibung wenig beachtete Unterklassen-Qualität der vormodernen Juden in Ost und West bezog, kann man darin den Ausdruck einer typisch absolutistischen ReformMentalität sehen. Aber die darin enthaltene Kritik am Judentum überdauerte die Vormoderne, verselbständigte sich: der Liberalismus eines Treitschke, überhaupt der Nationalliberalen drang nie zu einer Bejahung einer besonderen jüdischen Existenz durch. Die Gleichheit sollte vielmehr durch die Preisgabe der Eigenheiten, letztlich auch der Religion, erkauft werden. Die freie Entfaltung der Juden als Gruppe war nie ein echter Programmpunkt des Liberalismus.

Zur Frage des modernen Antisemitismus noch zwei weitere Hinweise. Die historische Antisemitismusforschung ist nicht unabhängig von den Ereignissen, die den Standpunkt des Historikers bestimmen. Die entscheidenden Ereignisse für unsere Generation sind der Nationalsozialismus, das Dritte Reich, der Holokaust. Man sieht Kontinuitäten, isoliert Stränge. Dann hält man die isolierten Stränge für die einzige Wirklichkeit. Dies gilt in besonderem Maß für die jüdische Geschichtsforschung, der ich mich zuzähle. Der Zwang, die Vergangenheit auf das blutige Ende hin zu ordnen, ist überwältigend und universal, er wird - vor allem in Israel und den USA - von einer breiten öffentlichen Meinung akzeptiert. Daneben gibt es langsam stärker werdende persönliche und institutionelle Neuansätze. Sie verlangen die Berücksichtigung zweier Tatsachen.

Erstens gab es ein durchaus positives Verhältnis von Juden und Christen, Juden und Deutschen in der Zeit der Emanzipation. Selbst wenn man auf die Grenzen hinweist bzw. sich die imbalances im Verhältnis von Majorität und Minorität bewußt macht, wie es etwa Gershom Scholem vor seinem Tod getan hat, bleibt die Tatsache einer überaus fruchtbaren Symbiose, einer überaus fruchtbaren Wechselwirkung in allen Bereichen. Die zweite Tatsache ist der nicht nur nationale, sondern europäische, ja, wenn man die USA einschließt, nicht einmal nur europäische Charakter der rassistischen, rassentheoretischen, nationalistischen, darwinistischen, elitetheoretischen Ideen, die zu Elementen des modernen Antisemitismus wurden. Die geistigen Einflüsse, die in den verschiedenen Ländern jeweils wirksam waren, waren vielfältig; die historischen Situationen waren verschieden. Ebenso war es der Stellenwert, der den bewußten Strömungen - etwa im Verhältnis zu vorhandenen Gegenströmungen - in der betreffenden politischen Kultur zukam. Die Dreyfus-Affäre in Frankreich resultierte in einem Sieg der liberalen Kräfte, einer Neu-Orientierung der kirchlichen Politik, wogegen alle Reform-Versuche des liberalen russischen Bürgertums scheiterten. Vergleiche der jeweils wirksamen Strukturen und Faktoren sind bisher 
nicht durchgeführt worden. Aber bei der Beschränkung der Betrachtung auf ein Land - hier: Deutschland - gilt es diesen internationalen Rahmen im Auge zu behalten.

Soweit zum modernen Antisemitismus in seiner historischen Epoche. Ich bin der Meinung, daß die beiden entscheidenden Ereignisse der jüngsten jüdischen Geschichte, der Massenmord an den Juden im Zweiten Weltkrieg und die Gründung des Staates Israel, einen qualitativen Bruch in der Geschichte des westlichen Antisemitismus bedeuten, so daß es gerechtfertigt ist, die Geschichte des traditionellen westlichen Antisemitismus mit der totalen Katastrophe des NS-Regimes und seiner Verbündeten und Satelliten in West- und Osteuropa zu Ende gehen zu lassen.

Diese Auffassung mag zunächst überraschend scheinen. Die dominierende Erneuerungstendenz in der Bundesrepublik Deutschland sah seit 1949 in der Bekämpfung des Antisemitismus eine Bewährungsprobe der liberal-demokratischen Ordnung, während man in der DDR in Äußerungen des Antisemitismus Symptome fortexistierender kapitalistischer und faschistischer Haltungen erblickte. Tatsächlich kam es - insofern waren jene Ansichten berechtigt - darauf an, die Verbrechen der Vergangenheit zu erkennen, die Verbrecher ihrer Strafe zuzuführen, neuen Anfängen zu wehren. Das Bestreben, das Wesen des Antisemitismus zu erkennen, um ein erneutes Aufleben rechtzeitig diagnostizieren, also vermeiden zu können, war auch eines der Motive für die Gründung des Berliner Zentrums für Antisemitismusforschung, das ich leite. Seine Arbeit beruht auf der Einsicht, daß die Werte der liberalen Demokratie unteilbar sind, daß also solche Forschung nicht nur für die jüdische Gemeinschaft, sondern vor allem auch für die deutsche Kultur notwendig ist, für eine entsprechende Gestaltung des Verhältnisses zu allen Minderheiten.

Was aber die vor allem für die jüdische Gemeinschaft wichtige Frage nach dem Stellenwert des zweifellos noch immer existierenden Antisemitismus betrifft, so hat der Holokaust das Zentrum des jüdischen Lebens demographisch und kulturell aus Ost- und Mitteleuropa nach den USA, Israel, England, Frankreich und anderen Ländern verlegt. Das zentrale Anliegen der organisierten jüdischen Gemeinschaften in diesen Ländern sind Bedingungen, die einerseits die Sicherheit Israels, seine Entwicklung zu einem friedlichen Kulturzentrum, andererseits die eigene Lebenskraft gewährleisten. Ein neues Aufleben des politischen Antisemitismus, des Antisemitismus als breite soziale Bewegung ist in der westlichen Welt in Anbetracht der Haltungen der politischen Eliten und der Diskreditierung extrem-totalitärer Herrschaftsformen in der vorhersehbaren Zukunft wenig wahrscheinlich. Und die Bundesrepublik Deutschland, deren Freundschaft für die jüdische Gemeinschaft - gerade auf Grund der Vergangen- 
heit, gerade wegen des vorhandenen guten Willens für die Zukunft - von besonderer Bedeutung ist, gehört zur westlichen Welt.

Dies zum Hintergrund, vor dem Antisemitismusforschung in einem Berliner Zentrum gegenwärtig möglich ist. Bemühungen um ein wissenschaftliches Verständnis des Antisemitismus setzten schon vor ca hundert Jahren ein, wenn man die ersten ernsteren Versuche des letzten Jahrzehnts des neunzehnten Jahrhunderts berücksichtigt. ${ }^{5}$ Seitdem ist die Literatur sehr gewachsen. Die antisemitische Seite selbst hat bis auf wenige Ausnahmen, zu denen auch einige im Dritten Reich entstandene Arbeiten gehören, wenig wissenschaftlich Brauchbares hervorgebracht. Die Werke von Walter Frank, Wilhelm Grau, Volkmar Eichstädt ${ }^{6}$ die zahlreichen Dissertationen, die an den deutschen Universitäten der Nazizeit geschrieben wurden, haben eher Quellenwert in bezug auf die ,,wissenschaftliche" Arbeit des Dritten Reichs als Aussagewert in bezug auf den Gegenstand selbst.

Die Antisemitismusforschung ist keine wertfreie Wissenschaft, kann es weder in Deutschland noch aus jüdischer Sicht sein. Sie beruht auf der Annahme, daß Antisemitismus zwar eine politische oder soziale Funktion haben kann, daß er unter Verwendung aller verfügbaren wissenschaftlichen Methoden in seinen verschiedenen Formen möglichst objektiv zu analysieren, in die allgemeine Geschichte einzuordnen ist, daß er aber in jedem Fall eine malfunction, sei es der Wahrnehmung, des emotionalen Haushalts von Individuen, sei es einer Gesellschaft, einer politischen Kultur, darstellt. Der Oberbegriff ist hier der der malfunction, die einer Verzerrung der Wirklichkeit Vorschub leistet, während die wissenschaftliche Forschung die Wirklichkeit so erfassen soll, daß das Ergebnis ein anhand der Folgen überprüfbares Modell darstellt.

In dem folgenden Überblick über die Grundformen des Antisemitismus bediene ich mich eines wissenschaftsgeschichtlichen Schemas, das erlauben wird, bei Vermeidung eines naiven Realismus den Kontakt zur Empirie zu wahren. Ich gehe so vor, daß ich die Darstellungen der verschiedenen Formen des Antisemitismus zunächst auf ihren wissenschaftlichen Ansatz

5 Vgl. H. Bahr, Der Antisemitismus. Ein internationales Interview, Berlin 1893; A. Bebel, Sozialdemokratie und Antisemitismus. Rede auf dem IV. Parteitag der Sozialdemokratischen Partei zu Köln a.Rh., Berlin 1894; B. Lazare, L'Antisémitisme. Son histoire et ses causes, Paris 1894.

${ }^{6} \mathrm{Vgl}$. H. Heiber, Walter Frank und sein Reichsinstitut für Geschichte des neuen Deutschlands, Stuttgart 1966, wo sich weitere Literaturhinweise finden, ferner u.a. W. Grau, Die Judenfrage als Aufgabe der neuen Geschichtsforschung, Hamburg [1935]; ders., Die Erforschung der Judenfrage. Aufgabe und Organisation, München [1943]; V. Eichstädt, Bibliographie zur Geschichte der Judenfrage, Bd 1: 1750-1848, Hamburg 1938. 
zurückführe, um dann das Modell im Lichte der historischen Erfahrung auf seine Brauchbarkeit für die Abwehr des Antisemitismus zu prüfen. Dabei stellt sich zunächst einmal die Frage nach der jeweiligen Form, dem - sit venia verbo - ontischen Status dessen, was zu beschreiben ist.

Die erste der Formen, die ich hier unterscheiden möchte, ist Gegenstand der Kulturanthropologie und Ethnologie; ihre historische Erforschung bedient sich der entsprechenden Methoden, vor allem der Methoden der Kultur- und Mentalitätsgeschichte. Es handelt sich um das Bild von Juden und Judentum, das sich in der Volkskultur des christlichen Europa, aber auch - in Übertragungen - in überseeischen Gebieten, etwa den nordamerikanischen, findet. Diese Form weist eine starke religiöse Komponente auf, die aus dem Paradox resultiert, daß ein Streit zweier jüdischer Sekten über die Nähe oder Ferne der Endzeit in die Vorstellungswelt des Christentums eindrang und spätestens seit den Kirchenvätern zu einem Topos der christlichen Überlieferung wurde. Diese Überlieferung hat m.E. auch die Ideenwelt des modernen Antisemitismus beeinflußt, jedenfalls aber dazu beigetragen, daß derselbe - auf Grund des Effekts des déjà vu, der den propagierten Ideen eine größere Wahrscheinlichkeit gab - so breite Unterstützung fand.

Es gibt eine große Zahl von Einzeluntersuchungen, die von diesem Ansatz ausgehen, aber keine zusammenfassende Darstellung. Das fragliche Bild des Juden findet sich in den Zeugnissen der Volksreligion, Predigten, Legenden, überlieferten Gerüchten (z.B. über sog. Ritualmorde); es findet sich in der religiösen Volkskunst, Passionsspielen, anderen rituellen Aufführungen; es begegnet uns in Volksmärchen, Volksliteratur, Pamphleten, Flugblättern, Zeitungsserien und ähnlichen Erzeugnissen, ferner in Bilderbögen, Karikaturen etc., wo das Bild des Juden vielfach häßliche, ja abstoßende Züge aufweist bzw. der Jude in abstoßenden Situationen dargestellt wird. Der Stürmer führte eine Tradition fort, die sich bis ins Mittelalter zurückverfolgen läßt. Das Problem entspricht dem aus den amerikanischen Rassenkonflikten bekannten Problem des ,Black is not beautiful".

Diese - von einem kulturanthropologischen Ansatz thematisierte-Form des Antisemitismus muß nicht offen hervortreten, sondern kann über lange Zeiträume hinweg latent existieren. Nichtsdestoweniger kommt ihr m.E. eine fundamentale Bedeutung zu, die bisher nur unzureichend erkannt wurde. Weder von jüdischer noch von nicht-jüdischer Seite wurde ihrer Abwehr dementsprechend die gebührende Beachtung geschenkt. Das könnte, was die jüdische Seite betrifft, u.a. darauf zurückzuführen sein, daß die Abwehr des Antisemitismus vorzugsweise in bürgerlich-intellektuellen Händen lag, bei Gruppen, die zumeist von Rechtsanwälten geführt 
wurden. Die Erkenntnis der tiefen Verankerung des negativen Bilds des Juden im Volksbewußtsein hätte das eigene Bewußtsein der erreichten Gleichheit in Frage gestellt. Außerdem waren die den bürgerlich-intellektuellen Kreisen angehörenden Juden nicht bereit, auf das Niveau herabzusteigen, auf dem eine wirksame Bekämpfung der fraglichen - für ,,vulgär" gehaltenen - Äußerungen möglich gewesen wäre. Besonders illustrieren dies die Diskussionen im Central-Verein deutscher Staatsbürger jüdischen Glaubens Anfang der dreißiger Jahre: die Verantwortlichen weigerten sich, auf das Niveau der Nazipropaganda - , in die Gosse" - herabzusteigen. ${ }^{7}$ Was die nicht-jüdische Seite betrifft, so sind die christlichen Kirchen erst nach 1945, auf Grund der Einsicht in ihr Versagen, dahin gelangt, in dem Antisemitismus und seinen Folgen auch eine Konsequenz christlicher Traditionen zu erblicken.

Das Entscheidende bei dieser Form des Antisemitismus ist, daß es sich dabei nicht um ein abgrenzbares Element in der Mentalität christlicher Gruppen handelt. Sie muß nicht eine deutliche Gestalt annehmen bzw. im Sinne des nächsten Ansatzes - zum Vorurteil werden.

Vom zweiten Ansatz aus stellt sich der Antisemitismus als ein gnoseologisches bzw. psychologisches Problem dar. Gemäß diesem wohl am häufigsten benutzten Ansatz beruht der Antisemitismus auf einem Vorurteil, also einer vorgefaßten Meinung, einer ,,falschen" Wahrnehmung. Die früheste, noch heute wirksame Fassung dieses Modells ist die rationalistische, die auf der Annahme basiert, daß die Welt hinsichtlich erkennbarer Tatsachen irrt. Die jüdische Gruppe ist in politischer, sozialer, wirtschaftlicher, religiöser Hinsicht in Wahrheit nicht das, wofür man sie hält. So gibt es in der Literatur (in der deutschen seit Lessing) das Bild des edlen Juden. Ebenso gibt es ein positives Judenbild in der Philosophie (in der deutschen seit Moses Mendelssohn), den Wissenschaften (es wird auf den deutschjüdischen Beitrag zu deren Entwicklung hingewiesen), der Kunst (hier drängt sich der Hinweis auf das kulturelle Leben der Weimarer Republik auf).

Ausgehend von dem jeweiligen Inhalt des Vorurteils, den Bestandteilen des jeweiligen Bilds des Juden, unterscheidet man einen religiösen, ökonomischen, politischen, rassischen, sonst einen Antisemitismus. Quellen zur ideengeschichtlichen Entwicklung dieser Varianten gibt es reichlich; die Literatur dazu ist uferlos. Es mag kraß klingen, aber als Ergebnis kann man festhalten, daß es in der westlichen Welt keine bedeutendere politische Ideologie, keine bedeutendere geistesgeschichtliche Bewegung gegeben

7 Vgl. u.a. A. Paucker, Der jüdische Abwehrkampf gegen Antisemitismus und Nationalsozialismus in den letzten Jahren der Weimarer Republik, 2. Aufl. . Hamburg 1969. 
hat, die nicht zur Entwicklung der antisemitischen Ideen beigetragen hätte. Das gilt für die Aufklärung wie für den Sozialismus, für die Romantik wie für den Liberalismus, für den Darwinismus wie für die davon abgeleiteten elitistischen Rassen- und Gesellschaftstheorien, etwa nach Art der von Georges Vacher de Lapouge. ${ }^{8}$

Schon das sollte zeigen, daß das rationalistische Modell des Vorurteils auf einer nicht-rationalen Grundlage beruhen muß; andernfalls wäre das universale Vorkommen des Vorurteils kaum zu erklären. Die notwendige Modifizierung des Modells wurde durch die neuere Vorurteilsforschung vorgenommen, die sich seit den dreißiger Jahren vor allem in den USA entwickelt hat. Der Ansatz ist in erster Linie individualpsychologisch, d.h. der Inhalt des Vorurteils wird unter dem Aspekt seiner Bedeutung für den, der es hegt, untersucht. Gewisse Ansatzpunkte bietet hier die Wahrnehmungspsychologie: das Vorurteil beruht auf Verallgemeinerungen von Teilbeobachtungen. Pars pro toto. Nur ein Teil der Wirklichkeit wird erkannt, aber dann absolut gesetzt. Das Vorurteil wird dementsprechend danach von der Wirklichkeit bis zu einem gewissen Grad bestätigt. Weitere Ansatzpunkte sind aus der Tiefenpsychologie gewonnen worden. Die fragliche Literatur ist i.d.R. wenig empirisch, meist werden Konsequenzen psychoanalytischer Konzepte entwickelt. Eine empirische Ausarbeitung wurde in einer Reihe versucht, deren bekanntestes Werk die berühmte Studie von Adorno u.a. über die Authoritarian Personality ist. ${ }^{9}$

Die Abwehr des antisemitischen Vorurteils bestand - und besteht noch immer - vorzugsweise in dem Bemühen um seine Korrektur. Dieses Bemühen illustriert namentlich die Geschichte des Central-Vereins deutscher Staatsbürger jüdischen Glaubens. Der Central-Verein blieb während seiner ganzen vierzigjährigen Tätigkeit - von 1893 bis 1933 - der rationalistischen Konzeption des Vorurteils verhaftet, selbst als ihm in der Endphase seines Kampfes gegen den Nationalsozialismus die Grenzen dieser Konzeption bewußt wurden. Es wäre reizvoll, seine Abwehrarbeit hier als einen paradigmatischen Fall, der Möglichkeiten und Grenzen der entsprechenden Tätigkeit einer Minderheit zeigt, ausführlicher darzustellen. Ich muß mich indessen auf ein paar Andeutungen beschränken. ${ }^{10}$

8 Vgl. G. Vacher de Lapouge, Les sélections sociales. Cours libre de science politique professé à l'Université de Montpellier (1888-1889), Paris o.J.; ders., L'aryen, son rôle social. Cours libre de science politique professé à l'Université de Montpellier (18891890), Paris 1899; ders., Race et milieu social. Essais d'anthroposociologie, Paris 1909. ${ }_{9}$ T. W. Adorno u.a., The Authoritarian Personality, New York [1950].

10 Vgl. Paucker, Der jüdische Abwehrkampf, a.a.O.; ferner ders., ,,Die Abwehr des Antisemitismus in den Jahren 1893-1933", in: Antisemitismus. Von der Judenfeindschaft zum Holocaust, hrsg. von H. A. Strauß und N. Kampe, Bonn [1984], S. 143-71 (ausführliche Bibliographie ebd., S. 164-71). 
Inmitten einer vom Liberalismus abrückenden Gesellschaft blieb der Central-Verein der liberalen Konzeption des Staats verpflichtet. Überdies begrenzte ihn von vornherein seine Auffassung, gemäß der die jüdische Gemeinschaft eine Religionsgemeinschaft deutscher Staatsbürger war bzw. sein sollte, in der Wahl der Mittel. Er beschränkte sich auf die klassischen Medien: Zeitung, Buch, Broschüre, öffentliche Versammlung. Jede ,Massenagitation” wurde abgelehnt. Erst in der letzten Zeit vor 1933 benutzte man auch verdeckte Kanäle, gezielte Nachrichtenversorgung, auf bestimmte Berufsgruppen und Multiplikatoren gerichtete Spezialliteratur.

Man hatte sich von vornherein gegen ein Auftreten als besondere politische Gruppe entschieden. Statt dessen arbeitete man mit den Parteien zusammen, die dazu bereit waren. Man trieb Honoratiorenpolitik. Bei der kleinen Zahl der Juden in Deutschland und bei ihrem Bild von sich als Deutschen - also nicht als Juden mit dem Anspruch auf Anerkennung als eigener Gruppe - kam eine Minderheitenpolitik kaum in Betracht. Nach 1930 wurde selbst diese schwache politische Aktivität noch reduziert; denn die Parteien wollten sich nicht mehr dem Vorwurf der ,,Judenknechtschaft" aussetzen, und es gelang den deutschen Juden nicht, sie davon zu überzeugen, daß die Gleichheit der Juden eigentlich eine deutsche, ihre eigene Sache, nämlich die ihres Selbstschutzes sei. So mußten sich die deutschen Juden vollständig mit dem System von Weimar identifizieren, dessen Untergang auch das Scheitern ihres Bemühens um die Abwehr des antisemitischen Vorurteils bedeutete.

Die deutschen Juden standen in der Weimarer Republik vor mannigfaltigen Dilemmas. Ihre Zahl war zu klein, um bei Wahlen ins Gewicht zu fallen. Das von der nationalistischen Propaganda verfälschte Bild der Juden war durch Teilerscheinungen geprägt: das Eintreten jüdischer Publizisten für einen Verständigungsfrieden im Ersten Weltkrieg; die Rolle vieler Juden in der Revolution 1918-19. Die Juden waren dadurch ihrerseits gezwungen, ihren Patriotismus durch den Hinweis auf die Anzahl der im Krieg gefallenen Juden zu beweisen. Jede Aufklärung erreichte aber nur die, die schon überzeugt waren. Gegen die breite, primitive, durch physische Gewaltanwendung unterstützte NS-Propaganda konnten die vom Central-Verein eingesetzten Mittel wenig ausrichten.

Der Erfahrung des Central-Vereins steht die amerikanische Erfahrung gegenüber. Es zeigt sich in den USA ein - in opinion polls festgestellter Rückgang antijüdischer Stereotypen und Vorurteile, eine zunehmend stabile Integration der Juden in das politische, wirtschaftliche und kulturelle Leben ihres Landes. Diese Entwicklung ist aber kaum der Abwehrarbeit zuzuschreiben, die - bei größerem Verständnis für Public Relations - im wesentlichen der des Central-Vereins entspricht. Vielmehr ist die Öffent- 
lichkeit - gemäß ihrem eigenen Selbstverständnis, das in nicht geringem Maß durch die historische Erfahrung der sukzessiven Integration immer neuer Einwanderungsschübe geprägt ist - einer liberalen Ideologie verpflichtet. Das zentrale Thema der Demokratisierung des Landes ist die Integration der schwarzen Bürger.

Ich bezweifle, daß die Aufklärungsarbeit der amerikanischen Bürgerrechtsorganisationen ohne den Kampf gegen das Dritte Reich, den Holokaust, die Gründung Israels, die tatsächliche Rolle der Juden in allen Bereichen des amerikanischen Lebens nach 1945 effektiv gewesen wäre. Der unbestreitbare Fortschritt im Verständnis des Antisemitismus, den der psychologische Ansatz gebracht hat, läßt sich kaum in Abwehrarbeit übersetzen, es sei denn durch eine psychologisch wirksamere Gestaltung der klassischen Aufklärungsarbeit. Die Möglichkeiten einer Gruppentherapie mit Antisemiten sind begrenzt. Antisemiten befinden sich kaum je, wenn überhaupt, in psychoanalytischer Behandlung.

Der dritte Ansatz ist der sozialgeschichtliche $b z w$. soziologische, von dem her sich der Antisemitismus als ein Problem von Gruppenbeziehungen darstellt. Das Interesse konzentriert sich auf die politischen und sozialen Entwicklungen in den Ländern, in denen Antisemitismus vorkam, sowie auf die Formen seiner Organisation und seines Wirkens. Es wird betont, daß der Antisemitismus in den betreffenden Gesellschaften - also etwa Deutschland seit 1875, Österreich seit der gleichen Zeit, Rußland seit 1881 , Frankreich zur Zeit der Dreyfus-Affäre - quasi eine symbolische Funktion erfüllt hat, derart, daß sein Anwachsen Probleme signalisiert, die nicht die waren, die er anzusprechen vorgab. Für das Deutsche Kaiserreich hat die Historiographie - besonders hingewiesen sei hier auf die Arbeiten von Eckart Kehr und Hans Rosenberg ${ }^{11}$ - die enge Bindung der jüdischen Emanzipationsbewegung an die liberale Bewegung herausgearbeitet. Als der Liberalismus zur Ideologie der Großindustrie wurde bzw. - in seiner fortschrittlich-demokratischen Variante - in der von Bismarck etablierten Ordnung seine Kraft verlor, wurden die deutschen Juden politisch heimatlos. Die enge Verbindung von liberaler und jüdischer Emanzipationsbewegung zeigt sich aber auch in Frankreich: der zunächst nur individuelle Fall eines zu Unrecht verurteilten Offiziers jüdischer Herkunft löste eine Konfrontation der Liberalen und ihrer linken Verbündeten, zu denen sich nach einigem Zögern auch Jean Jaurès und Jules Guesde samt ihrem sozialistischen Anhang gesellten, mit den ultrakonservativen Kräften in Armee und

$"$ Vgl. E. Kehr, Der Primat der Innenpolitik. Gesammelte Aufsätze zur preußischdeutschen Sozialgeschichte im 19. und 20. Jahrhundert, hrsg. und eingel. von H.-U. Wehler, Berlin 1965; H. Rosenberg, Große Depression und Bismarckzeit. Wirtschaftsablauf, Gesellschaft und Politik in Mitteleuropa, Berlin 1967. 
Kirche aus. In Rußland wurde zum Symbol einer ähnlichen Konstellation die Fälschung, die unter dem Namen "Protokolle der Weisen von Zion" zur Bibel des internationalen Antisemitismus avancierte. Ihren Kern bildet der dann auch von Hitler kolportierte Vorwurf, daß die Juden etwa die Ideologie des Liberalismus nur benutzten, um ihre Weltherrschaft zu sichern. Der Kampf gegen das Judentum wurde so zum Kampf um die heiligsten Güter der Nation, die Ordnung, den abendländischen Geist. In Österreich wiederum ist der Antisemitismus in die Nationalitäten- und sozialen Konflikte nach dem Ausgleich von 1867 einzuordnen; erinnert sei hier an die Ursprünge des Nationalsozialismus in der deutsch-tschechischen Konfrontation, den Pan-Germanismus Schönerers, die kleinbürgerlich-katholische Bewegung des Bürgermeisters Lueger in Wien.

Die fragliche Literatur führt zu dem Schluß, daß sich die Gesellschaft jeweils in einer Krise befand, in der interessierte Schichten und Gruppen den Antisemitismus als Integrationssymbol benutzten. Für Deutschland hat Rosenberg in seiner Arbeit über die große Depression den Aufschwung der antisemitischen Bewegung zu dem Abschwung der Kondratieff-Welle von 1873 bis 1895 in Beziehung gesetzt. Durch eine Reihe Einzeluntersuchungen ist diese These in der Folge beträchtlich verfeinert worden. Aber das Modell bleibt das eines Spannungszustands, der seine Ursache in malfunctions und Verwerfungen der wirtschaftlichen und sozialen Entwicklung hat.

Eine wichtige, aber bisher nur wenig ausgearbeitete Modifizierung dieses Modells besteht in der Einbeziehung der jüdischen Gruppe selbst in diesen Spannungszustand. So geht etwa Eva Reichmann, die wieder auf die Notwendigkeit dieser Einbeziehung hingewiesen hat, davon aus, daß es eine ,objektive Judenfrage" gab. ${ }^{12}$ Der Antisemitismus resultiert nach dieser Sicht aus objektiven Spannungen zwischen Juden und Umwelt, Minderheit und Mehrheit. Die jüdische Gemeinschaft spielte wirklich eine

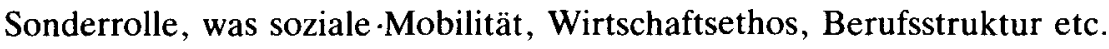
betraf. Es gab soziale Gruppen, die sich tatsächlich bedroht fühlen bzw. tatsächlich jüdische Aktivitäten als ,,schädlich" erfahren konnten. Der Antisemitismus war auch der Ausdruck dieser objektiven Spannungen.

Dieser Ansatz berührt sich mit dem von Marx schon 1843 formulierten Vorwurf, daß Juden als die eigentlichen Vertreter des Kapitalismus zu gelten hätten und samt dem Kapitalismus der sozialen Revolution zum Opfer fallen würden. Eine Variante dieser Sicht ist die von Karl Kautsky und den Austro-Marxisten Otto Bauer und Karl Renner vertretene Auffas-

12 E. G. Reichmann, Hostages of Civilization. The social sources of National Socialist anti-semitism, London 1950. 
sung, daß die Juden als Gruppe keine Merkmale aufwiesen, die sie zu einer Nation machten, und mit der Überwindung des kapitalistischen Systems verschwinden würden - eine Auffassung, die im Grunde von Ablehnung bzw. Selbsthaß zeugt. Die spätere orthodox-marxistische Deutung des Antisemitismus, etwa in dem einzigen Buch darüber, das vor 1933 von einem Kommunisten geschrieben wurde, ${ }^{13}$ weicht nicht wesentlich von dieser Linie ab. Die marxistische Nachkriegsliteratur zum Faschismusproblem hat den Antisemitismus in ihre Faschismustheorie eingefügt und ihm derart eine Eigengesetzlichkeit abgesprochen. Er gilt als ideologisches Manipulationsinstrument, dessen sich der Kapitalismus in seiner Herrschaftsausübung bedient, oder, wie es ein amerikanischer Autor ausgedrückt hat, als ,,red herring". ${ }^{14}$ Andere marxistische Autoren suchen immer noch die Ermordung der Juden - im Gegensatz zu Tim Masons These vom Primat politischer Motive - auf ökonomische Ursachen zurückzuführen. ${ }^{15}$

Wenn der Antisemitismus - gemäß dem sozialgeschichtlichen Ansatz in seinen verschiedenen Varianten - das Ergebnis gesellschaftlicher Strukturen ist, dann sind die Möglichkeiten der direkten Abwehr recht begrenzt. Weder ihrer Zahl noch ihrer Berufsstruktur nach hatten die Juden in irgendeinem Land West- oder Osteuropas eine ausschlaggebende Bedeutung, derart, daß sie die Strukturen, die den Antisemitismus bewirkt hätten, ihrerseits hätten beeinflussen können. Selbst wo die These von der Abnormalität der eigenen Gruppe von den Juden selbst akzeptiert wurde, brachte die etwas großartig als ,,jüdische Sozialpolitik" bezeichnete Reformtätigkeit nur marginale Resultate. Nur die Verbindung von Verfolgung, Emigration und Siedlung in Israel hat für einen Teil des deutschen Judentums zu einer einschneidenden Änderung der Berufsstruktur geführt. Aber sie war ohne Einfluß auf die Entwicklung des Antisemitismus, die in der Vernichtung des europaïschen Judentums kulminierte.

Abschließend will ich noch auf zwei Problemkomplexe hinweisen.

Es stellt sich die Frage, inwieweit die Antisemitismusforschung das Modell bestätigen kann, das sich für Deutschland aus der oben angesprochenen Wendung der Geschichtsschreibung von - um es einmal schlagwortartig auszudrücken - dem Primat der Außenpolitik zu dem der

13 Vgl. O. Heller, Der Untergang des Judentums, Berlin 1931.

${ }_{14} \mathrm{Vgl}$. E. Weber, Varieties of Fascism. Doctrines of revolution in the twentieth century, Princeton, N.J. [1964], S. 62-69.

is Vgl. T. Mason, ,,Der Primat der Politik - Politik und Wirtschaft im Nationalsozialismus", in: Das Argument, Jg. 8 (1966), S. 473-84; E. Czichon, ,, Der Primat der Industrie im Kartell der nationalsozialistischen Macht", ebd., Jg. 10 (1968), S. 168-92; T. Mason, ,,Primat der Industrie? - Eine Erwiderung”, ebd., S. 193-209. 
Innenpolitik ergibt. Wir hören viel von einer Korrektur der Vorstellung von einem deutschen Sonderweg, die einer ,,Tendenzwende" in der Geschichtsschreibung gleichkäme, nach einem neuen Modell verlange. Die vergleichende Forschung bestätigt und modifiziert jene Interpretation. Sie bestätigt sie, insofern das Modell, das das Anwachsen des Antisemitismus in Deutschland - zunächst im Kaiserreich, dann nach 1930 - zu erklären sucht, seine Fruchtbarkeit ebenso für andere westliche Länder, aber auch für Rußland und Polen bewiesen hat. Sie modifiziert jene Interpretation, insofern die konkreten politischen Entscheidungen, militärische Ereignisse, die Außenpolitik qua Motiv der Innenpolitik die in dem strukturgeschichtlichen Modell zusammengefaßten Tatsachenreihen jeweils verändert haben. Insofern ist der Antisemitismus in jedem Land in einen Sonderweg einzubetten. Die Vorzüge des englischen bzw. amerikanischen Modells der Modernisierung, wie sie Geoff Eley und David Blackbourn - reklamieren, ${ }^{16}$ stehen zur Diskussion; was den Antisemitismus betrifft, scheint mir der Ausgang dieser Diskussion belanglos.

Das zweite Problem ist das des neuen Antisemitismus, wie er sich in der Benutzung antisemitischer Motive in der außenpolitischen Diskussion um die Politik Israels sowie in der auf innenpolitische Ziele abgestimmten Propaganda kommunistischer Länder kundtut. Was das zweite Phänomen betrifft, so dürften zur Erklärung - mutatis mutandis - vorstehend skizzierte Ansätze verwendbar sein. Die Propaganda, die auf ein - sei es tatsächlich vorhandenes, sei es unterstelltes - antisemitisches Potential einspielt, entspricht den gerade von marxistischer Seite betonten Manipulationstendenzen herrschender Eliten. Das Problem der Abwehr dieses neuen Antisemitismus ist noch ungeklärt. Es erfordert ein weiteres Durchdenken der Frage von eventuellen Kontinuitäten, macht aber auch unmittelbar bewußt, daß die Entwicklung in ein neues Stadium eingetreten ist. Verändert hat sich insbesondere die Zielsetzung der Abwehr: es geht in der nach-emanzipatorischen Epoche nicht mehr um die Gleichberechtigung einzelner Juden, sondern um die Sicherstellung der jüdischen Gruppenexistenz. Sie verlangt ein politisches Konzept der Abwehr. Unabhängig von den aktuellen Problemen der Nahostpolitik dürfte sich auch in der neuen Epoche das Paradigma der liberalen Tradition wertvoll erweisen: die Gesundheit einer Gesellschaft - bzw. des Welt-Systems - mißt sich an der Weise, in der mit den Minderheiten - bzw. kleinen Ländern - umgegangen wird.

16 G. Eley, Reshaping the German Right. Radical nationalism and political change after Bismarck, New Haven, Conn., London 1980; D. Blackbourn, Class, Religion and Local Politics in Wilhelmine Germany. The Centre Party in Württemberg before 1914, Wiesbaden 1980 . 